\title{
Visual perception in prediagnostic and early stage Huntington's disease
}

\author{
BRIAN F. O'DONNELL, ${ }^{1}$ TANYA M. BLEKHER,${ }^{2}$ MARJORIE WEAVER,${ }^{3}$ KERRY M. WHITE, ${ }^{3}$ \\ JEANINE MARSHALL, ${ }^{3}$ XABIER BERISTAIN,${ }^{4}$ JULIE C. STOUT, ${ }^{1}$ JACQUELINE GRAY, ${ }^{3}$ \\ JOANNE M. WOJCIESZEK,${ }^{4}$ AND TATIANA M. FOROUD ${ }^{3}$ \\ ${ }^{1}$ Department of Psychological and Brain Sciences, Indiana University, Bloomington, Indiana \\ ${ }^{2}$ Department of Ophthalmology, Indiana University School of Medicine, Indianapolis, Indiana \\ ${ }^{3}$ Department of Medical and Molecular Genetics, Indiana University School of Medicine, Indianapolis, Indiana \\ ${ }^{4}$ Department of Neurology, Indiana University School of Medicine, Indianapolis, Indiana
}

(Received June 18, 2007; Final Revision December 14, 2007; Accepted December 18, 2007)

\begin{abstract}
Disturbances of visual perception frequently accompany neurodegenerative disorders but have been little studied in Huntington's disease (HD) gene carriers. We used psychophysical tests to assess visual perception among individuals in the prediagnostic and early stages of HD. The sample comprised four groups, which included 201 nongene carriers (NG), 32 prediagnostic gene carriers with minimal neurological abnormalities (PD1); 20 prediagnostic gene carriers with moderate neurological abnormalities (PD2), and 36 gene carriers with diagnosed HD. Contrast sensitivity for stationary and moving sinusoidal gratings, and tests of form and motion discrimination, were used to probe different visual pathways. Patients with HD showed impaired contrast sensitivity for moving gratings. For one of the three contrast sensitivity tests, the prediagnostic gene carriers with greater neurological abnormality (PD2) also had impaired performance as compared with NG. These findings suggest that early stage HD disrupts visual functions associated with the magnocellular pathway. However, these changes are only observed in individuals diagnosed with HD or who are in the more symptomatic stages of prediagnostic HD. (JINS, 2008, 14, 446-453.)
\end{abstract}

Keywords: Huntington's disease, Visual perception, Visual pathways, Neurodegenerative disease, Neuropsychology, Contrast sensitivity

\section{INTRODUCTION}

Huntington's disease (HD) is an autosomal dominant disorder resulting from an increased number of triplet (CAG) repeats in the Huntington gene (Huntington's Disease Collaborative Research Group, 1993). Whereas clinical and neuropsychological studies have documented marked dysfunction of motor and cognitive functions in HD (Ho et al., 2003), several lines of evidence suggest that visual pathways may also be affected in this disorder. Patients with HD exhibit visual evoked potential abnormalities (Ellenberger et al., 1978; Oepen et al., 1981) indicative of retinostriate dysfunction. Structural MRI assessment has shown reductions of grey matter (Fennema-Notestine et al., 2004;

Correspondence and reprint requests to: Brian F. O'Donnell, Department of Psychological and Brain Sciences, Indiana University, 1101 E. $10^{\text {th }}$ Street, Bloomington, IN 47405. E-mail: bodonnel@indiana.edu
Mühlau et al., 2007; Rosas et al., 2002) and white matter in the occipital cortex (Beglinger et al., 2005; FennemaNotestine et al., 2004). Post-mortem increases in gammaaminobutyric acid (GABA) concentrations in the cortex, with the largest increases in the striate cortex, have also been reported (Storey et al., 1992). Visual perceptual impairments have been reported in other neurodegenerative disorders. For example, patients with Parkinson's disease show deficits on tests of contrast sensitivity (Rodnitzky, 1998; Uc et al., 2005), color vision (Rodnitzky, 1998), and motion perception (Uc et al., 2005). In Alzheimer's disease, deficits in contrast sensitivity (Gilmore et al., 2005; Rizzo et al., 2002) and motion perception (Rizzo et al., 2002) have been described. These findings suggest an evaluation of early stage vision in HD is warranted.

Disturbances in perception of visual cognition for stationary stimuli have frequently reported in this disorder 
(e.g. Gómez-Tortosa et al., 1996; Mohr et al., 1991), but there is a paucity of investigations which use psychophysical tests to evaluate perception of specific visual features. Psychophysical tests measure visual performance thresholds as a function of such factors as contrast, noise, stimulus duration, or stimulus similarity. Methodologically, psychophysical tests using staircase procedures allow estimation of performance thresholds for different visual features at the same difficulty level within and across subjects. In a pilot study using staircase procedures, O'Donnell and colleagues (2003) found that eight subjects with recently diagnosed HD demonstrated disturbances in motion perception, with intact contrast sensitivity for stationary stimuli. Festa et al. (2005), on the other hand, did not detect a motion perception deficit in a small sample of six HD patients. A larger sample of HD patients would provide a more definitive answer to this question, and inclusion of prediagnostic gene carriers would clarify whether such disturbances predate diagnosis.

To ensure sufficient power to detect deficits in specific visual pathways prior to diagnosis, the present study evaluated a larger sample of individuals at-risk for HD than O'Donnell et al. (2003). An expanded set of visual tests used psychophysical techniques to obtain performance thresholds for stimuli, which probed different visual pathways. Visual pathways within the central nervous system are specialized for different aspects of the visual environment. In primates and humans, two neural pathways for visual processing have been distinguished in terms of the response properties of the magnocellular $(\mathrm{M})$ and parvocellular $(\mathrm{P})$ neurons of the lateral geniculate nucleus (Livingstone \& Hubel, 1988; Wandell, 1995). The M pathway is characterized by high temporal resolution, low spatial resolution, and insensitivity to color. The P pathway has low temporal resolution, high spatial resolution, and strong color opponency responses. Functional differentiation continues into the cortex, with regional specialization for motion, color, faces, spatial relationships, and other visual features (Wandell, 1999). For example, the human homolog to MT, a region specialized for motion perception, has been localized to occipital-parietal and occipital-temporal cortical regions (Vaina et al., 2001). Object and facial recognition is carried out within the ventral pathway projecting from the occipital to inferior temporal cortex.

In the present study, contrast sensitivity for a high spatial frequency, stationary grating was used to test the P pathway. Moving low spatial frequency gratings were used to test the M pathway. Discrimination of dot motion trajectory in noise was used to assess cortical regions specialized for motion processing, including the human homolog of MT. Form discrimination in noise tested the ventral pathway. Based on our pilot study (O'Donnell et al., 2003), we predicted that gene carriers would show impairments on tests which probed the magnocellular and dorsal pathways: contrast sensitivity for moving gratings and dot motion discrimination. By comparing gene carriers who differed in their proximity to diagnosis, the sensitivity of these tests of visual perception to the neurodegenerative process was evaluated. Proximity to clinical onset was characterized by neurologic evaluation and by an algorithm based CAG length (Langbehn et al., 2004).

\section{METHODS}

\section{Participants}

Participants were recruited through the National Research Roster for Huntington's disease patients and families, a registry of patients and families interested in participating in research. Inclusion criteria were: (1) a parent with HD; (2) either undiagnosed, or diagnosed with HD within the past 2 years; (3) between the ages of 19 and 65; and (4) absence of other neurological illness or a major psychiatric disorder. All participants had normal or corrected visual acuity (20/40 or better) on a Snellen Test of near vision. $87 \%$ of subjects had acuity values of $20 / 25$ or better, and acuity not differ among groups (Fisher Exact Test $p$ value $=.53$ ). This study was approved by the Indiana University School of Medicine Institutional Review Board (Protocol 0109-12), conformed to ethical standards of the 1964 Declaration of Helsinki, and all participants provided informed consent. Demographic and clinical information from the study participants $(n=289)$ are summarized in Table 1 . There were no significant differences in age, education or gender distribution between the four groups.

All subjects were administered the Unified Huntington's Disease Rating Scale (UHDRS), a standardized clinical evaluation (Huntington Study Group, 1996). The motor portion of the UHDRS was administered by an experienced movement disorder neurologist, who was aware that the participant was at-risk for HD but was blind to their gene status and results of other assessments during the visit. As part of the UHDRS, the neurologist assigned a confidence rating using a scale of 0 to 4 to indicate the extent of motor abnormalities and the neurologist's confidence that the abnormalities represented symptoms of HD. A score of 0 indicated the evaluation was normal (no abnormalities). A score of 1 represented non-specific motor abnormalities with a confidence of less than $50 \%$ that these indicated a diagnosis of HD. A score of 2 indicated motor abnormalities that may be signs of HD with a confidence of 50-89\%. A score of 3 suggested the motor abnormalities were likely signs of HD with a confidence of $90 \%$ to $98 \%$. A score of 4 indicated the motor abnormalities were unequivocal signs of HD with a confidence in the diagnosis of $\geq 99 \%$. In addition, UHDRS composite scores for overall motor impairment (motor assessment questions 1 to 15), chorea (question 12), and ocular motor impairment (questions 1 to 3) were separately evaluated. The Digit Symbol Test (Wechsler, 1981), a neuropsychological measure with demonstrated sensitivity to early cognitive changes in gene carriers (Foroud et al., 1995; Kirkwood et al., 2000), was also administered. Digit Symbol is a multifactorial test, which taps psychomotor speed, attention, and working memory mechanisms. The age scaled 
Table 1. Demographic, clinical, and neuropsychological characteristics

\begin{tabular}{lccccc}
\hline \hline & NG & PD1 & PD2 & HD & \\
Characteristic & $N=201$ & $N=32$ & $N=20$ & $N=36$ & $p$ value \\
\hline Age $(y)$ & $47(10)$ & $45(10)$ & $45(12)$ & $47(11)$ & .60 \\
Education (y) & $15(2)$ & $16(2)$ & $16(2)$ & $16(3)$ & .59 \\
Gender & $56 \mathrm{M} / 145 \mathrm{~F}$ & $11 \mathrm{M} / 21 \mathrm{~F}$ & $7 \mathrm{M} / 13 \mathrm{~F}$ & $15 \mathrm{M} / 21 \mathrm{~F}$ & .34 \\
CAG Repeats & $\mathrm{N} / \mathrm{A}$ & $42(2)$ & $42(2)$ & $43(3)+$ & .02 \\
UHDRS Motor & $4.8(3.7)$ & $5.2(3.3)$ & $14.4(6.3)^{*}$ & $26.8(10.2)^{*}$ & $<.0001$ \\
UHDRS Ocular Motor & $1.3(1.9)$ & $1.2(1.8)$ & $4.9(2.6)^{*}$ & $5.6(2.8)^{*}$ & $<.0001$ \\
UHDRS Chorea & $.2(.7)$ & $.3(.9)$ & $2.6(2.8)^{*}$ & $8.2(4.1)^{*}$ & $<.0001$ \\
Digit Symbol & $11.8(2.4)$ & $11.2(2.9)^{*}$ & $10.5(2.7)^{*}$ & $8.9(2.5)^{*}$ & .0001 \\
\hline \hline
\end{tabular}

Note. Entries indicate mean values and SDs in parentheses for each group, with the exception of gender. ANOVAs were used to test for main effects of group for education, UHDRS scores, CAG repeats, and Digit Symbol. Gender was evaluated using Fisher's exact test statistic. For ANOVAs, $p$ values indicate an effect of group. Asterisks indicate differences between the non-gene carrier (NGC) group and gene carrier group (PD1, PD2, HD). The "+" symbol indicates that the HD group differed from the PD1 and PD2 groups $(p<.02$ in both cases). The Digit Symbol test score was age scaled.

score of the WAIS-R version of the test was used to allow comparison with our previous studies.

All subjects provided either a blood or a buccal swab sample, which was used for DNA extraction. Molecular testing was performed to determine the number of CAG repeats in the Huntington gene (Bond \& Hodes, 1996). Results from the molecular testing and the neurological evaluation were combined to assign each subject to a unique group. Nongene carriers (NG) were those subjects with two unexpanded ( $<32$ CAG repeats) HD alleles. Prediagnostic gene carrier group 1 (PD1) included those subjects with an expanded ( $\geq 38$ CAG repeats) HD allele and a UHDRS confidence rating of 0 or 1 . Prediagnostic gene carrier group 2 (PD2) consisted of participants with an expanded HD allele and an overall rating on the UHDRS of 2 or 3. Those subjects with an expanded HD allele who had an overall rating on the UHDRS of 4 were classified as manifest HD (HD). For gene carriers, time to diagnosis was estimated using the equation: time to diagnosis $=(21.54+\operatorname{EXP}(9.56-0.146 *$ CAGrepeats)) - (age at testing) based on analysis by Langbehn et al. (2004). Estimated time to diagnosis is the difference between a subject's estimated age of diagnosis and age at the time of the study visit.

\section{Visual Testing Procedures}

Participants were seated in a dark room with the subject's forehead $70 \mathrm{~cm}$ from the CRT display. Head restraint was not used. Testing was performed by trained study staff that were blind to the genetic and neurological status of the participants. Testing instructions and practice trials were given prior to administration of each test. In order to minimize motor demands, subjects responded verbally and staff entered each response on the computer.

\section{Contrast sensitivity tests}

Contrast sensitivity was evaluated using stationary and moving gratings. In the stationary grating detection task, a sinu- soidal grating with a spatial frequency of 9.9 cycles/degree of visual angle served as the target stimulus for a duration of $1000 \mathrm{~ms}$. The grating was initially presented at $42 \%$ Michelson contrast. Tone pips signaled the onset and offset of the trial. Fifty percent of the trials presented a grating, and $50 \%$ were null trials. The subject indicated whether a grating was present or not.

In the moving grating discrimination task, a sinusoidal grating with a spatial frequency of 1.3 cycles/degree was temporally modulated to produce motion to the right or left at one of three temporal frequencies $(2.1,9.3$, and 18.8 cycles/sec) for $480 \mathrm{~ms}$. The grating was initially presented at $30 \%$ Michelson contrast. Tone pips signaled the onset and offset of each trial. After the offset of each stimulus, the subject responded whether the grating appeared to move to the right or to the left.

For the stationary and moving grating tasks, contrast was varied in steps of $0.05 \mathrm{log}$ units and a staircase procedure was used to estimate $75 \%$ correct threshold level (O'Donnell et al., 2003; Tyler \& McBride, 1995). For all tasks, $\log _{10}$ contrast sensitivity was used for statistical analysis. Higher contrast sensitivity values indicated better performance.

\section{Form and motion discrimination tests}

Perception of form and dot motion properties were evaluated using psychophysical tests (Brenner et al., 2003; O'Donnell et al., 2006). In the form discrimination task, subjects were required to discriminate between two shapes (circle vs. square with rounded corners) at different levels of static visual noise. Stimuli were displayed with $75 \times 75$ pixel window, which subtended 2.9 degrees of visual angle. Each stimulus was presented for three seconds. Noise was added by randomly assigning a percentage of pixels within the display to black or white values. In order to quickly find the threshold range, the size of the noise increment was initially $13.3 \%$. After the first error the increment was reduced to $6.7 \%$, and after the further error the increment was reduced to $2.7 \%$. 
The dot motion trajectory discrimination task consisted of a field of dots moving either right or left across the screen at a velocity of 3.5 degrees/second. One hundred dots were presented for $500 \mathrm{~ms}$ in a rectangular display subtending 8.1 degrees visual angle. Thresholds were obtained by varying the number of dots that were moving randomly (dynamic visual noise). As in the form discrimination condition, the size of the increment in noise was initially large at $10 \%$. After an error trial, the increment was $5 \%$, and after the fourth error, the increment was reduced to $2 \%$.

Both tasks used an adaptive staircase method to estimate performance thresholds (Levitt, 1971; O'Donnell et al., 2006). The subject responded verbally to each trial. The subject's performance gradually converged around a visual noise threshold value, which was the amount of noise required to obtain a $71 \%$ performance level. The visual noise threshold, calculated as the mean value of the final six trials of the staircase was the dependent measure used for analysis (O'Donnell et al., 2006). Higher visual noise thresholds indicate better performance.

\section{Statistical Analysis}

The distribution of each visual test variable was reviewed to assess normality and detect extreme outliers defined as greater than 4 standard deviations from the mean. These extreme values were assigned as missing values for the remaining analyses. One-way analysis of variance (ANOVA) was then performed to detect group effects (NG, PD1, PD2, HD) for the six tests of visual perception, and UHDRS scores. ANCOVA with gender and age as co-variates was used to compare Digit Symbol scores. For those measures demonstrating a significant group effect, one-sided $t$-tests were used in post-hoc analyses to evaluate which of the three gene carrier groups differed significantly from the NG group. Fisher Exact Test was used to evaluate categorical data. Pearson correlation coefficients were used to test relationships between clinical measures and visual perception tests. All subjects had complete neurological and genetic data. Subjects with missing data for a perceptual or neuropsychological measure were excluded from analysis including that measure.

\section{RESULTS}

\section{Visual Tests}

Significant group effects were observed for three of the six tests of visual perception (Table 2). Significant group effects were found with all three contrast sensitivity tests using moving gratings at the $2.1 \mathrm{~Hz}$ modulation rate $(F(3,278)=$ $14.8, p<.0001), 9.3 \mathrm{~Hz}(F(3,279)=8.5, p \leq .0001)$ and $18.8 \mathrm{~Hz}(F(3,279)=3.4, p=.02)$. Post-hoc analyses demonstrated that the individuals with manifest $\mathrm{HD}$, even though they were in the very early stages of illness, performed significantly worse than the NG and PD1 groups on all three tests $(p<.01)$, whereas the difference between the HD and PD2 groups was only significant for gratings at $2.1 \mathrm{~Hz}(p=.001)$. Post-hoc analyses to identify abnormalities among the two prediagnostic gene carrier groups and nongene carriers only identified significant differences when comparing the PD2 and NG groups on gratings at $9.3 \mathrm{~Hz}$ $(p=.01)$, whereas no significant differences were observed between the PD1 and NG groups. The PD2 group performed worse than the PD1 group for the $9.3 \mathrm{~Hz}(p=.01)$ moving gratings. Significant group effects were not observed for contrast sensitivity with the stationary grating. Noise thresholds for form discrimination and dot motion discrimination did not differ among groups.

\section{Clinical Measures}

UHDRS total motor $(p<.0001)$, chorea $(p<.0001)$, and ocular motor $(p<.0001)$ scores all showed differences among groups. In all cases, HD and PD2 groups were impaired compared to the NG group, whereas the PD1 group did not differ from NG. ANCOVA employing the Digit Symbol Test detected a significant group effect $(F(5,278)=$ $13.3, p<.0001)$. Post-hoc comparisons of each of the gene

Table 2. Mean scores for visual perception thresholds

\begin{tabular}{lcccc}
\hline \hline & NG & PD1 & PD2 & HD \\
Test & $N=201$ & $N=32$ & $N=20$ & $N=36$ \\
\hline Contrast sensitivity & & & & \\
$\quad$ Stationary & $1.79(.36)$ & $1.68(.33)$ & $1.78(.39)$ & $1.73(.42)$ \\
$\quad$ 2.1 Hz modulation & $2.09(.13)$ & $2.10(.13)$ & $2.05(.14)$ & $1.92(.19)^{*}$ \\
$9.3 \mathrm{~Hz}$ modulation & $2.13(.13)$ & $2.14(.13)$ & $2.05(.18)^{*}$ & $2.02(.19)^{*}$ \\
$\quad$ 18.8 Hz modulation & $1.68(.19)$ & $1.73(.15)$ & $1.64(.14)$ & $1.59(.25)^{*}$ \\
Discrimination thresholds & & & & \\
$\quad$ Dot motion & $81.8(9.2)$ & $79.5(12.1)$ & $80.7(9.1)$ & $77.1(10.6)$ \\
$\quad$ Form & $71.9(9.1)$ & $74.3(10.1)$ & $73.1(9.6)$ & $69.1(13.9)$ \\
\hline \hline
\end{tabular}

Note. Entries indicate mean values and SDs in parentheses for each group. Contrast sensitivity indicates the $\log _{10}$ contrast sensitivity (1/contrast threshold). Higher values indicate better performance. Form and motion thresholds indicate percent noise at threshold. Higher thresholds indicate better performance. *Post-hoc comparison with NG significant at $p<.05$. 
carrier groups with the NG group were performed, and all comparisons were significant (NG vs. PD1: $p=0.04$; NG vs. PD2: $p=0.008$; NG vs. HD: $p<0.0001)$. These data are shown in Table 1.

\section{Correlation Analysis}

Estimated time to diagnosis, but not the number of CAG repeats, was correlated with the thresholds for moving gratings (Table 3). These results provide further evidence of the sensitivity of moving grating contrast sensitivity to proximity of onset of the disorder. Among the neuropsychological tests, Digit Symbol was correlated with a single visual test measure, contrast sensitivity at the $2.1 \mathrm{~Hz}$ modulation rate.

\section{DISCUSSION}

The present study indicated that patients with HD show a selective deficit in contrast sensitivity for moving gratings relatively early in the neurodegenerative process. This deficit was present in gene carriers who were closest to onset based on neurological examination and CAG repeats. This may represent a selective deficit in the symptomatic gene carriers compared to non-gene carriers, since other measures of visual function did not differ significantly among groups.

Contrast sensitivity for moving gratings was impaired for individuals with manifest HD. In addition, gene carriers in the PD2 group who showed non-specific motor abnormalities were also impaired on one of these tests. These specific abnormalities suggest selective dysfunction in the magnocellular (M) pathway, which is sensitive to low spatial frequencies, high temporal frequencies, and luminance (Livingstone \& Hubel, 1988; Wandell, 1995). Stationary grating contrast sensitivity was unaffected in any of the gene carrier groups, suggesting that the parvocellular $(\mathrm{P})$ pathway, which prefers medium to high spatial frequencies and low temporal frequencies, is less sensitive to the early effects of HD. Similarly, previous studies of HD patients have not detected deficits for contrast sensitivity utilizing stationary gratings (O'Donnell et al., 2003; Sprengelmeyer et al., 1996). The spared high spatial frequency contrast sensitivity found in HD carriers differs from several other clinical conditions, suggesting that this deficit may be related to specific neuropathological changes in HD. For example, aging is associated with relatively spared low spatial frequency, and impaired high spatial frequency contrast sensitivity for stationary gratings (Spear, 1993). Contrast sensitivity deficits in Parkinson's disease vary with stage of illness and severity but appear most pronounced at intermediate spatial frequencies (Rodnitzky, 1998). In a study of patients with schizophrenia, Slaghuis (1998) found that patients diagnosed with schizophrenia were impaired for moving and stationary gratings, with most consistent deficits at higher spatial frequencies. Unlike the present HD sample, patients diagnosed with Parkinson's disease (Uc et al., 2005) and schizophrenia (O'Donnell et al., 2006) can show dot motion deficits as well as impaired stationary contrast sensitivity. Thus, in Parkinson's disease, aging, and schizophrenia, the pattern of visual deficits frequently differs from that of HD gene carriers.

These disturbances in contrast sensitivity could be produced by a variety of neuropathological mechanisms. Magnocellular pathway abnormalities could occur in the retina, lateral geniculate nuclei of the thalamus, or occipital cortex. Whereas detailed neuropathology of the visual pathways is not yet available in HD, visual evoked potential abnormalities in HD have been described in diagnosed patients, suggestive of retinostriate or visual cortex dysfunction (Ellenberger et al., 1978; Oepen et al., 1981). Structural MRI findings have directly implicated abnormalities of gray and white matter in visual cortex. Several MRI reports indicate that white matter volume is reduced in the cerebral cortex (Beglinger et al., 2005), and that white matter volume reduction may be most severe in the occipital cortex in HD patients (Beglinger et al., 2005; FennemaNotestine et al., 2004). With respect to gray matter volume, some reports indicate widespread reduction in volume, including the occipital cortex (Fennema-Notestine et al., 2004; Mühlau et al., 2007), although other investigators have reported no reduction in gray matter volumes (Beglinger et al., 2005). Rosas et al. (2002) reported MR measured thinning of the cortical ribbon, which was most severe in posterior brain regions, including dorsal visual cortex (Brodmann areas 17, 18, and 19). Storey et al. (1992) reported an increase in gamma-aminobutyric acid (GABA) concentrations in multiple cortical regions, with the largest increases in striate cortex (area 17). GABAergic neurons seem to play an important role in visual processing in ani-

Table 3. Correlations among perceptual, genetic, and neuropsychological variables in gene carriers

\begin{tabular}{lrrrrrrr}
\hline \hline & \multicolumn{2}{c}{ Visual test } & & \multicolumn{3}{c}{ Grating (Modulation rate) } \\
\cline { 2 - 3 } \cline { 5 - 7 } Measure & Form & Motion & & (Static) & $(2.1)$ & $(9.3)$ & $(18.8)$ \\
\hline$C A G$ & .02 & -0.09 & & .06 & -.11 & .05 & .07 \\
Est. Time to Diagnosis & .20 & .06 & & .18 & $.34^{* *}$ & $.24^{*}$ & $.34^{* *}$ \\
Digit Symbol & .13 & .04 & & -.06 & $.25^{*}$ & .14 & .07 \\
\hline \hline
\end{tabular}

Note: CAG refers to number of CAG repeats. Est. Time to Diagnosis refers to the estimated years to diagnosis based on number of CAG repeats and age at the time of testing. $* * p<.01 ; * p<.05$. 
mals (Egelhaaf et al., 1990; Leventhal et al., 2003) and humans (Giersch \& Herzog, 2004). Abnormalities in posterior cortical visual systems could contribute to disturbances in eye movements and motion perception. Functional MRI, for example, suggests that the lateral occipitotemporal region is activated during perception of moving gratings and visual pursuit of a moving dot (Barton et al., 1996). In conjunction, the present behavioral data and pathophysiological data from other studies suggest that detailed analysis of retinothalamic and cortical visual pathways may reveal early changes in gene carriers.

Tests of form and motion discrimination suggested that noise thresholds for these processes were not affected in the gene carriers, similar to findings by Festa et al. (2005). However, in O'Donnell et al. (2003) diagnosed HD patients did show impairments on a test of dot motion discrimination. This difference is most likely caused by differences in severity between the HD samples in these two studies. In the present study, the mean scaled Digit Symbol score in the HD group was 8.9, suggesting mild impairment. In the O'Donnell et al. (2003) sample, on the other hand, the mean age adjusted Digit Symbol score of the HD group was 5.0, suggestive of marked impairment nearly 2 SD below the control group mean. In summary, these results suggest that the ventral and dorsal cortical pathways may not be affected in the early stages of the disease.

Among the functional measures used in this study, the Digit Symbol was the most sensitive to the presence of the HD genotype. It was the only test significantly impaired in both prediagnostic gene carrier groups as well as the HD group. The magnitude of the deficit, even in the diagnosed HD group, was less than one SD, indicating that symptomatic gene carriers were at an early stage of the disease process. The sensitivity of the Digit Symbol Test in prediagnostic gene carriers replicates findings from some (Foroud et al., 1995; Kirkwood et al., 2000) but not all studies (HahnBarma et al., 1998). These differences are most likely because of variations in CAG repeats and age between samples, because these are strongly related to the proximity to the onset of the clinical disorder (Jason et al., 1997). For example, on a test of motor timing variability, Hinton et al. (2007) found that estimated years to onset of the illness was correlated with motor timing variability in prediagnostic gene carriers.

Several issues arise in interpreting differential deficits on vision tests. Tests that vary in difficulty may also vary in sensitivity to behavioral deficits. In order to minimize this possibility, the psychophysical staircase method estimates thresholds at specific difficulty levels and thereby match performance on difficulty within and between subjects. For the contrast sensitivity tests, thresholds were estimated at a difficulty level of $75 \%$, and for the noise tests of dot motion and form perception, thresholds were estimated at $72 \%$. Thus, thresholds were obtained within a very narrow range of difficulty. Secondly, the display time of the moving grating tests was shorter than the stationary grating test. Because HD is associated with psychomotor slowing, the shorter display time could have contributed to the deficit on this task. However, only one visual test measure correlated with Digit Symbol, suggesting that that visual perceptual performance does not simply indicate overall level of intellectual impairment or psychomotor slowing.

The results from the present data and other studies suggest that the neurodegenerative process in HD proceeds in a nonparallel fashion with respect to visual processing. During the prediagnostic period (PD1) when gene carriers show minimal neurological abnormality, deficits have been observed in some neuropsychological tests such as Digit Symbol (Foroud et al., 1995; Kirkwood et al., 2000), in quantitative saccades (Blekher et al., 2006), and in motor timing variability (Hinton et al., 2007). In contrast, the selective deficit for moving, low frequency gratings observed in this study was only detected in the more symptomatic prediagnostic gene carriers (PD2) and in individuals with manifest HD. The present study, and studies by O'Donnell et al. (2003) and Sprengelmeyer et al. (1996), found that contrast sensitivity for stationary gratings was unaffected in the early stages of HD. Disturbances in visual cognition for stationary stimuli in the absence of memory demands has been most consistently found in diagnosed HD patients, suggesting that these functions may be mildly affected in the earliest stages of the illness (Gómez-Tortosa et al., 1996; Jacobs et al., 1995; Mohr et al., 1991; Sprengelmeyer et al., 1996).

In summary, gene carriers late in the prediagnostic stage of HD and patients with diagnosed HD showed a deficit in contrast sensitivity for temporally modulated gratings. Among gene carriers, contrast sensitivity was correlated with estimated time to onset. With respect to the neuropathology of HD, these data implicate involvement of the prestriate visual pathways or cortical visual pathways relatively early in the disease process. Use of structural and functional neuroimaging in conjunction with behavioral testing could help identify the neural correlates of these abnormalities in visual perception. These data further suggest that the neurodegenerative process in HD does not proceed in a parallel fashion, and have important implications when considering which biomarkers would be most effective for use in studies of pharmacological agents.

\section{ACKNOWLEDGMENTS}

The authors thank the participants in this study. Dr. Christopher W. Tyler of the Smith Kettlewell Eye Institute, San Francisco, CA assisted with the contrast sensitivity methods. This study was supported by NINDS RO1 NS42659 (Foroud), N01-NS-2326 (Foroud), and NIMH 1 RO1 MH62150-01 (O’Donnell).

\section{REFERENCES}

Barton, J.J., Simpson, T., Kiriakopoulos, E., Stewart, C., Crawley, A., Guthrie, B., Wood, M., \& Mikulis, D. (1996). Functional MRI of lateral occipitotemporal cortex during pursuit and motion perception. Annals of Neurology, 40, 387-398.

Beglinger, L.J., Nopoulos, P.C., Jorge, R.E., Lanbehn, D.R., Mikos, A.E., Moser, D.J., Duff, K., Robinson, R.G., \& Paulson, J.S. 
(2005). White matter volume and cognitive dysfunction in early Huntington's disease. Cognitive and Behavioral Neurology, 18, $102-107$.

Blekher, T., Johnson, S.A., Marshall, J., White, K., Hui, S., Weaver, M., Gray, J., Yee, R., Stout, J.C., Beristain, X., Wojcieszek, J., $\&$ Foroud, T. (2006). Saccades in presymptomatic and early stages of Huntington disease. Neurology, 67, 394-399.

Bond, C. \& Hodes, M.E. (1996). Direct amplification of the CAG Repeat of Huntington without amplification of CCG. Clinical Chemistry, 42, 773-774.

Brenner, C.A., Wilt, M.A., Lysaker, P.H., \& O’Donnell, B.F. (2003). Psychometrically matched tasks of discrimination and recognition performance in schizophrenia spectrum disorders. Journal of Abnormal Psychology, 112, 28-37.

Egelhaaf, M., Borst, A., \& Pilz, B. (1990). The role of GABA in detecting visual motion. Brain Research, 509, 156-160.

Ellenberger, J.C., Petro, D.J. \& Ziegler, S.B. (1978). The visual evoked potentials in Huntington disease. Neurology, 28, 95-97.

Fennema-Notestine, C., Archibald, S.L., Jacobson, M.W., CoreyBloom, C., Paulsen, J.S., Peavy, G.M., Gamst, A.C., Hamilton, J.M., Salmon, D.P., \& Jernigan, T.L. (2004). In vivo evidence of cerebellar atrophy and cerebral white matter loss in Huntington disease. Neurology, 63, 989-995.

Festa, E.K., Insler, R.Z., Salmon, D.P., Paxton, J., Hamilton, J.M., \& Heindel, W.C. (2005). Neocortical disconnectivity disrupts sensory integration in Alzheimer's disease. Neuropsychology, $19,728-738$.

Foroud, T., Siemers, E., Kleindorfer, D., Bill, D.J., Hodes, M.E., Norton, J.A., Conneally, P.M., \& Christian, J.C. (1995). Cognitive scores in carriers of Huntington's disease gene compared to noncarriers. Annals of Neurology, 37, 657-664.

Giersch, A. \& Herzog, M.H. (2004). Lorazepam strongly prolongs visual information processing. Neuropsychopharmacology, 29, 1386-1394.

Gilmore, G.C., Cronin-Golomb, A., Neargarder, S.A., \& Morrison, S.R. (2005). Enhanced stimulus contrast normalizes visual processing of rapidly presented letters in Alzheimer's disease. Vision Research, 45, 1013-1020.

Gómez-Tortosa, E., del Barrio, A., Barroso, T., \& Garcia Ruiz, P.J. (1996). Visual processing disorders in patients with Huntington's disease and asymptomatic carriers. Journal of Neurology, 243, 286-292.

Hahn-Barma, V., Deweer, B., Dürr, A., Dodé, C., Feingold, J., Pillon, B., Agid, Y., Brice, A., \& Dubois, B. (1998). Are cognitive changes the first symptoms of Huntington's disease? A study of gene carriers. Journal of Neurology, Neurosurgery and Psychiatry, 64, 172-177.

Hinton, S.C., Paulsen, J.S., Hoffmann, R.G., Reynolds, N.C., Zimbelman, J.L., \& Rao, S.M. (2007). Motor timing variability increases in preclinical Huntington's disease as estimate onset of motor symptoms approaches. Journal of the International Neuropsychological Society, 13, 539-543.

Ho, A.K., Sahakian, B.J., Brown, R.G., Barker, R.A., Hodges, J.R., Ané, M.N., Snowden, J., Thompson, J., Esmonde, T., Gentry, R., Moore, J.W., Bodner, T., \& NEST-HD Consortium. (2003). Profile of cognitive progression in early Huntington's disease. Neurology, 61, 1702-1706.

Huntington Study Group. (1996). Unified Huntington's Disease Rating Scale: Reliability and consistency. Movement Disorders, 11, 136-142.

Huntington's Disease Collaborative Research Group. (1993). A novel gene containing a trinucleotide repeat that is expanded and unstable on Huntington's disease chromosomes. Cell, 72, 971-983.

Jacobs, D.H., Shuren, J., \& Heilman, K.M. (1995). Impaired perception of facial identity and facial affect in Huntington's disease. Neurology, 45, 1217-1218.

Jason, G.W., Suchowersky, O., Pajurkova, E.M., Graham, L., Klimek, M.L., Garber, A.T., \& Poirier-Heine, D. (1997). Cognitive manifestations of Huntington disease in relation to genetic structure and clinical onset. Archives of Neurology, 54, 1081-1088.

Kirkwood, S.C., Siemers, E., Hodes, M.E., Conneally, P.M., Christian, J.C., \& Foroud, T. (2000). Subtle changes among presymptomatic Huntington's disease mutation carriers. Journal of Neurology Neurosurgery and Psychiatry, 69, 773-779.

Langbehn, D.R., Brinkman, R.R., Falush, D., Paulsen, J.S., \& Hayden, M.R. (2004). International Huntington's Disease Collaborative Group. A new model for prediction of the age of onset and penetrance for Huntington's disease based on CAG length. Clinical Genetics, 65, 267-277.

Leventhal, A.G., Wang, Y., Pu, M., Zhou, Y., \& Ma, Y. (2003). GABA and its agonists improve visual cortical function in senescent monkeys. Science, 300, 812-815.

Levitt, H. (1971). Transformed up-down methods in psychoacoustics. Journal of the Acoustical Society of America, 49, 467-477.

Livingstone, M. \& Hubel, D. (1988). Segregation of form, color, movement and depth: Anatomy, physiology and perception. Science, 240, 740-749.

Mohr, E., Brouwers, P., Claus, J.J., Mann, U.M., Fedio, P., \& Chase, T. (1991). Visuospatial cognition in Huntington's disease. Movement Disorders, 6, 127-132.

Mühlau, M., Weindl, A., Wohlschläger, A.M., Gaser, C., Städtler, M., Valet, M., Zimmer, C., Kassubek, J., \& Peinemann, A. (2007). Voxel-based morphometry indicates relative preservation of the limbic prefrontal cortex in early Huntington disease. Journal of Neural Transmission, 114, 367-372

O’Donnell, B.F., Bismark, A., Hetrick, W.P., Bodkins, M., Vohs, J.L., \& Shekhar, A. (2006). Early stage vision in schizophrenia and schizotypal personality disorder. Schizophrenia Research, 86, 89-98.

O’Donnell, B.F., Wilt, M.A., Hake, A., Stout, J.C., Kirkwood, S.C., \& Foroud, T. (2003). Visual function in Huntington's disease patients and presymptomatic mutation carriers. Movement Disorders, 18, 1027-1034.

Oepen, G., Doerr, M., \& Thoden, U. (1981). Visual (VEP) and somatosensory (SSEP) evoked potentials is Huntington's chorea. Electroencephalography and Clinical Neurophysiology, 51, 666-670.

Rizzo, M., Anderson, S.W., Dawson, J., \& Nawrot, M. (2002). Vision and cognition in Alzheimer's disease. Neuropsychologia, 38, 157-169.

Rodnitzky, R.L. (1998). Visual dysfunction in Parkinson's disease. Clinical Neuroscience, 5, 102-106.

Rosas, H.D., Liu, A.K., Hersch, S., Glessner, M., Ferrante, R.J., Salat, D.H., van der Kouwe, A., Jenkins, B.G., Dale, A.M., \& Fischl, B. (2002). Regional and progressive thinning of the cortical ribbon in Huntington's disease. Neurology, 58, 695-701.

Slaghuis, W.L. (1998). Contrast sensitivity for stationary and drifting spatial frequency gratings in positive- and negativesymptom schizophrenia. Journal of Abnormal Psychology, 107, 49-62. 
Spear, P.D. (1993). Neural bases of visual deficits during aging. Vision Research, 33, 2589-2609.

Sprengelmeyer, R., Young, A.W., Calder, A.J., Karnat, A., Lange, H., Hömberg, V., Perrett, D.I., \& Rowland, D. (1996). Loss of disgust: Perception of faces and emotions in Huntington's disease. Brain, 119, 1647-1665.

Storey, E., Kowall, N.W., Finn, S.F., Mazurek, M.F., \& Beal, M.F. (1992). The cortical lesion of Huntington's disease: Further neurochemical characterization, and reproduction of some of the histological and neurochemical features by N-methyl-Daspartate lesions of rat cortex. Annals of Neurology, 32, 526-534.

Tyler, C.W. \& McBride, B. (1995). The Morphonome Image Psychophysics Software and Calibrator for Macintosh Systems. Spatial Vision, 10, 479-484.
Uc, E.Y., Rizzo, M., Anderson, S.W., Qian, S., Rodnitzky, R.L., \& Dawson, J.D. (2005). Visual dysfunction in Parkinson disease without dementia. Neurology, 65, 1907-1913.

Vaina, L.M., Cowey, A., Eskew, R.T. Jr., LeMay, M., \& Kemper, T. (2001). Regional cerebral correlates of global motion perception: Evidence from unilateral cerebral brain damage. Brain, 124, 310-321.

Wandell, B.A. (1995). Foundations of vision. Sunderland, MA: Sinauer.

Wandell, B.A. (1999). Computational neuroimaging of human visual cortex. Annual Review of Neuroscience, 22, 145-173.

Wechsler, D. (1981). Wechsler Adult Intelligence Scale-Revised. New York: Psychological Corp. 